\title{
Indicadores antropométricos e hemodinâmicos de risco cardiovascular e fatores associados à pressão arterial elevada em mineradores ${ }^{1}$
}

\author{
Marcelo Magalhães Sales ${ }^{a}$, Thales Boaventura Rachid Nascimento ${ }^{b}$, Ricardo Yukio Asano ${ }^{c}$, \\ José Fernando Vila Nova de Moraes ${ }^{\mathrm{d}}$, Rafael da Costa Sotero ${ }^{\mathrm{e}}$, \\ Verusca Najara de Carvalho Cunha ${ }^{e}$, Rodrigo Alberto Vieira Browne ${ }^{\mathrm{f}}$, Herbert Gustavo Simóes ${ }^{\mathrm{g}}$
}

\author{
aDoutorando em Educação Física, Universidade Católica de Brasília - UCB, Taguatinga, DF, Brasil \\ ${ }^{b}$ Mestre em Educação Física, Universidade Católica de Brasília - UCB, Taguatinga, DF, Brasil \\ 'Doutorando em Educação Física, Universidade Católica de Brasília - UCB, Taguatinga, DF, Brasil \\ Professor Adjunto, Centro Universitário Regional de Gurupi - UNIRG, Gurupi, TO, Brasil \\ ${ }^{\mathrm{d}}$ Doutorando em Educação Física, Universidade Católica de Brasília - UCB, Taguatinga, DF, Brasil. \\ Professor Assistente, Universidade Federal do Vale do São Francisco - UNIVASF, Petrolina, PE, Brasil \\ eDoutorandos em Educação Física e Professores Assistentes, \\ Universidade Católica de Brasília - UCB, Taguatinga, DF, Brasil \\ ${ }^{\mathrm{f}}$ Mestrando em Educação Física, Universidade Federal do Rio Grande do Norte - UFRN, Natal, RN, Brasil \\ gDoutor em Ciências Fisiológicas, Universidade Federal de São Carlos - UFSCar, São Carlos, SP, Brasil \\ Professor Titular e Diretor do Programa de Pós-graduação Stricto Sensu em Educação Física, \\ Universidade Católica de Brasília - UCB, Taguatinga, DF, Brasil
}

\begin{abstract}
Resumo: A obesidade e a hipertensão arterial são tidas como fatores de risco para doenças cardiovasculares e a sua prevalência parece diferir de acordo com a atividade profissional. Desse modo, o objetivo do presente estudo foi estimar a prevalência de fatores de risco cardiovascular em funcionários de uma mineradora e verificar os fatores associados à pressão arterial (PA) elevada. Para tanto, 197 voluntários (61,4\% homens) com idade entre 20 e 60 anos, todos funcionários de uma empresa de mineração, foram avaliados quanto ao índice de massa corporal e PA. O excesso de peso (sobrepeso + obesidade) e os valores pressóricos elevados (pré-hipertenso + hipertenso) foram observados em $76,1 \%$ e $46,1 \%$ da amostra, respectivamente, sendo a prevalência de pré-hipertensos maior ( $\mathrm{p}<0,05)$ nos homens. A probabilidade de ter PA elevada foi maior no sexo masculino (11\%), e naqueles com idade superior a 40 anos (13\%) e com excesso de peso (13\%). Em conclusão, uma parcela expressiva dos mineradores encontra-se em risco para o desenvolvimento de doenças cardiovasculares. Ademais, as medidas contra a hipertensão arterial devem ser direcionadas especialmente aos funcionários do sexo masculino e aqueles com idade superior a 40 anos, sendo a prevenção e o controle do excesso de peso as principais medidas terapêuticas a ser adotadas.
\end{abstract}

Palavras-chave: Hipertensão, Obesidade, Doenças Cardiovasculares, Mineração.

\section{Anthropometric and hemodynamic indicators of cardiovascular risk and associated factor with high blood pressure in miners}

\begin{abstract}
Obesity and hypertension are risk factors for cardiovascular diseases and their prevalence seems to vary according to profession. In this scenario, the aim of this study was to estimate the prevalence of cardiovascular risk factors in employees of a mining company and to identify associated factors with high blood pressure
\end{abstract}

Autor para correspondência: Marcelo Magalhães Sales, Programa de Pós-graduação Stricto Sensu em Educação Física, Universidade Católica de Brasília, EPTC, QS 07, LT 1, bloco G, sala 116, Águas Claras, CEP 72022-900, Taguatinga, DF, Brasil, e-mail: marcelomagalhaessales@gmail.com

Recebido em 4/10/2012; Revisão em 3/1/2013; Aceito em 18/1/2013. 
(BP). To this end, 197 volunteers (61.4\% male) aged between 20 and 60, all employees of a mining company had their body mass index and BP measured. Excessive weight (overweight + obesity) and high BP values (pre-hypertension + hypertension) were observed in $76.1 \%$ and $46.1 \%$ of the sample, respectively; and the prevalence of pre-hypertensive individuals was higher $(p<0.05)$ in men. The likelihood of having high BP was higher in males (11\%), in those above than 40 years old (13\%) and overweight (13\%). In conclusion, a significant portion of the miners assessed is at risk for developing cardiovascular disease. Furthermore, measures against hypertension must be directed to male employees and those above than 40 years old. Overweight prevention and control are the main therapeutic measures to be adopted.

Keywords: Hypertension, Obesity, Cardiovascular Diseases, Miners.

\section{Introdução}

A obesidade é tida como um grave problema de saúde pública em países de alta e média renda (POPKIN; DOAK, 1998), e sua presença está associada ao surgimento do diabetes mellitus tipo 2, doenças cardíacas, síndrome metabólica, apneia do sono, osteoartrose, hipertensão (BRAY, 2004) e até mesmo a diversos tipos de cânceres (PAN et al., 2004). Por ser uma doença crônica e multifatorial, a gêneses da obesidade se dá por meio da interação de fatores metabólicos (VERMA et al., 2008), genéticos (RANKINEN et al., 2006) e principalmente ambientais (LAHTI-KOSKI et al., 2002).

No que se refere à hipertensão arterial, esta parece ser o principal fator de risco de morte entre as doenças crônicas não transmissíveis (CHOBANIAN et al., 2003), e a sua presença está associada ao desenvolvimento de doenças cardiovasculares (WANG et al., 2006). Tão determinante é a pressão arterial que a mortalidade por doença vascular se eleva com valores pressóricos maiores que $115 / 75 \mathrm{mmHg}$ (LEWINGTON et al., 2002). A idade, a obesidade (WANG et al., 2006) e os níveis de estresse (MARTINEZ; LATORRE, 2006) são fatores associados à hipertensão arterial.

A obesidade e a hipertensão parecem estar relacionadas com a atividade profissional, uma vez que a presença desses fatores difere entre as profissōes (MARTINEZ; LATORRE, 2006; MOREIRA et al., 2011; CAVAGIONI; PIERIN， 2010; QUEIROGA et al., 2009; CASSANI et al., 2009; MOLENA-FERNANDES et al., 2006). No setor de seguro-saúde (MOLENA-FERNANDES et al., 2006) e na indústria alimentícia (QUEIROGA et al., 2009), a obesidade e a hipertensão arterial acometem menos de $10 \%$ dos funcionários. Por outro lado, ambos os fatores de risco são observados em mais de um terço dos motoristas profissionais (CAVAGIONI; PIERIN, 2010).
No Brasil, a produção e os investimentos no setor mineral vêm exibindo comportamento crescente ao longo dos anos (INSTITUTO..., 2011), o que acarreta em aumento no número de profissionais atuantes nesse setor. Apesar disso, não foi encontrado nenhum estudo nacional que tenha investigado a presença de fatores de risco para doenças cardiovasculares em profissionais da área de mineraçáo.

Conhecer a distribuiçáo e os elementos associados aos fatores de risco das doenças cardiovasculares é essencial para elaboração e direcionamento das medidas preventivas contra esse tipo de doença; além disso, cada atividade profissional exibe suas peculiaridades.

Diante do exposto, o presente estudo teve como objetivo estimar a prevalência de fatores de risco antropométricos e hemodinâmicos para doenças cardiovasculares em mineradores bem como verificar os fatores associados à pressão arterial elevada.

\section{Material e métodos}

Estudo observacional e de corte transversal foi realizado em Oriximiná, Pará, Brasil, no ano de 2011. O município apresenta 62.794 habitantes (INSTITUTO..., 2010a) e índice de desenvolvimento humano de 0,72 (PROGRAMA..., 2012). A amostra foi recrutada no distrito de Porto Trombetas, ao qual pertence, e está localizado a $70 \mathrm{~km}$ de Oriximiná. Todos os indivíduos (370 indivíduos) engajados na atividade de mineraçáo do referido distrito na época (2011) foram convidados pelo pesquisador responsável para participar do estudo por meio de uma minipalestra (15 minutos), realizada durante o período de refeiçáo intrajornada (30 minutos) previsto para os trabalhadores dessa categoria.

Os critérios de inclusão foram: ter idade igual ou superior a 20 anos; residir há mais de um ano no referido distrito; estar diretamente engajado na atividade de mineração. Foram excluídos aqueles que 
não concordaram em participar do estudo ou que não atendiam aos critérios de inclusão. Onze indivíduos foram excluídos da análise por estarem há menos de um ano trabalhando na atividade de mineração. Os outros 162 indivíduos restantes não compareceram para a realização das coletas durante o período (2 dias) em que os pesquisadores estiveram presentes no referido distrito. Dessa maneira, participaram do estudo 197 mineradores $(53,24 \%$ da população investigada), sendo 121 homens e 76 mulheres, com idade entre 20 e 60 anos.

Antes da coleta de dados, os voluntários foram esclarecidos acerca dos procedimentos os quais seriam submetidos e assinaram um termo de consentimento livre e esclarecido, conforme Resolução 196/96 do Conselho Nacional de Saúde do Brasil (BRASIL, 1996). Em seguida, os voluntários tiveram, primeiramente, suas variáveis antropométricas mesuradas e por último as hemodinâmicas.

\subsection{Medidas antropométricas}

A massa corporal foi mensurada por meio de uma balança digital (Toledo 2096 PP), com resolução e capacidade de 0,05 e $200 \mathrm{~kg}$, respectivamente. $\mathrm{O}$ índice de massa corporal (IMC) foi calculado considerando-se o quociente entre a massa corporal (Toledo 2096 PP) em quilogramas e a estatura em metros (estadiômetro SECA ${ }^{\oplus} 214$, USA) elevada à segunda potência $\left(\mathrm{kg} \cdot \mathrm{m}^{-2}\right)$.

Os voluntários foram classificados (ORGANIZAÇÃO..., 1995) em baixo peso (IMC < 18,5 kg.m $\left.{ }^{-2}\right)$, eutrófico $\left(18,5-24,5 \mathrm{~kg} \cdot \mathrm{m}^{-2}\right)$, sobrepeso $\left(25,0-29,9 \mathrm{~kg} \cdot \mathrm{m}^{-2}\right)$ e obesidade $\left(>30,0 \mathrm{~kg} \cdot \mathrm{m}^{-2}\right)$. Para as análises de associação, a amostra foi categorizada em dois grupos: eutrófico e excesso de peso (sobrepeso + obesidade).

\subsection{Medidas hemodinâmicas}

As medidas de pressão arterial foram realizadas por meio de um aparelho automático (Microlife, BP 3AC1-1), após repouso de 5 min em ambiente calmo e tranquilo. Para tanto, os indivíduos permaneceram na posição sentada, pernas descruzadas, pés apoiados no chão, dorso recostado na cadeira e relaxado. O braço posicionado na altura do coração (nível do ponto médio do esterno ou quarto espaço intercostal), apoiado com a palma da mão voltada para cima e o cotovelo ligeiramente fletido, conforme procedimentos previamente descritos (SOCIEDADE..., 2010). Os indivíduos foram classificados conforme sugerido por Chobanian et al. (2003). A pressão arterial média (PAM) foi calculada:
PAM = pressão arterial diastólica $+1 / 3$ (pressão arterial sistólica - pressão arterial diastólica). Para as análises de associação, a amostra foi categorizada em dois grupos: normotenso e pressão arterial elevada (pré-hipertenso + hipertenso).

\subsection{Tratamento estatístico}

A estatística descritiva e o teste de KolmogorovSmirnov foram utilizados para caracterizar a amostra e verificar a normalidade dos dados, respectivamente. Para a comparação das médias e das proporçôes entre homens e mulheres foram utilizados o teste $t$ de student náo pareado e teste qui-quadrado, respectivamente. A associação da pressão arterial elevada (desfecho) com o sexo, idade e IMC foi verificada por meio da regressão de Poisson, com ajuste robusto para variância. Além disso, o poder estatístico a priori de todos os procedimentos utilizados (teste $t$ de student não pareado, qui-quadrado e regressão de Poisson) foi analisado. O poder estatístico a priori conferido à comparação entre sexos (teste $t$ de student não pareado) foi de $95 \%$, com um alfa de $5 \%$ para análise e um effect size de $\mathrm{r}=0,3$. Para a análise de comparação entre proporçôes (qui-quadrado), o poder estatístico calculado foi de $92 \%$, considerando um alfa de 5\% e um effect size de $\mathrm{w}=0,3$. Da mesma forma para análise de associação (regressão de Poisson), em que o effect size calculado foi de $95 \%$, quando inserido na análise um alfa de $5 \%$, um effect size $\operatorname{de} f=0,15$, além de três variáveis preditoras (sexo, idade e IMC). Os dados foram analisados por meio do software Statistical Package for the Social Sciences (SPSS) 15.0 e Gpower 3.0.10 adotando-se um nível de significância de $5 \%(\mathrm{p}<0,05)$.

\section{Resultados}

Os homens apresentaram valores superiores $(\mathrm{p}<0,05)$ às mulheres para a idade $(40,1 \pm 9,0$ vs $34,4 \pm 8,1)$, IMC $(28,80 \pm 4,2$ vs $27,18 \pm 4,5)$, PAS $(125,5 \pm 19,1$ vs $113,3 \pm 16,1)$, PAD $(78,9 \pm 12,1$ vs $72,6 \pm 12,6)$ e PAM $(94,4 \pm 14,0$ vs $82,6 \pm 13,2)$ (Tabela 1).

O excesso de peso (sobrepeso + obesidade) foi observado em 76,1\% da amostra, sem diferença entre os sexos, sendo que $49,6 \%$ e $33,1 \%$ dos homens foram classificados como sobrepesados e obesos, respectivamente (Tabela 2). Da mesma maneira as mulheres, que apresentaram prevalência de sobrepeso de $40,8 \%$ e obesidade de $25 \%$ (Tabela 2).

No que se refere à pressão arterial, os valores pressóricos elevados (pré-hipertenso + hipertenso) foram observados em $46,1 \%$ dos voluntários, sendo 
a prevalência de pré-hipertensão maior $(\mathrm{p}<0,05)$ nos homens (40,5\%) do que nas mulheres $(23,7 \%)$ (Tabela 2). Não foram observadas diferenças significativas $(\mathrm{p}>0,05)$ entre os sexos para o estrato hipertenso, sendo que $14,9 \%$ e $9 \%$ dos homens e mulheres, respectivamente, foram classificados no referido estrato.

A PA elevada (desfecho) associou-se ( $\mathrm{p}<0,05)$, na análise bruta, com todas as variáveis, sendo que os homens demonstraram possuir maior chance (17\%) de apresentar PA elevada quando comparado a seus pares do sexo feminino. Da mesma forma quando a amostra foi categorizada por idade e adiposidade corporal, ou seja, indivíduos que possuem idade > 40 anos e excesso de peso (sobrepesado + obeso) demonstram chance $17 \%$ maior de apresentarem PA elevada quando comparado aos indivíduos classificados com idade $\leq 40$ anos e eutróficos (IMC $\left.<25 \mathrm{~kg} \cdot \mathrm{m}^{-2}\right)$. Na análise ajustada, as associações se mantiveram $(\mathrm{p}<0,05)$, com o sexo masculino, a idade $>40$ anos e o excesso de peso, conferindo probabilidade $11 \%$, $13 \%$ e $13 \%$ maior de ter PA elevada, respectivamente (Tabela 3).

\section{Discussão}

A obesidade (BRAY, 2004) e a hipertensão arterial (WANG et al., 2006) são fatores de risco para doenças cardiovasculares e a sua prevalência parece diferir de acordo com a atividade profissional (MARTINEZ; LATORRE, 2006; MOREIRA et al., 2011; CAVAGIONI; PIERIN, 2010; QUEIROGA et al., 2009; CASSANI et al., 2009; MOLENA-FERNANDES et al., 2006). Embora a atividade de mineração seja uma profissão em ascensão (INSTITUTO..., 2011), nenhum estudo descreveu a prevalência de fatores de risco para doenças cardiovasculares nesses profissionais. Desse modo, objetivou-se estimar a prevalência de fatores de risco antropométricos e hemodinâmicos para doenças cardiovasculares em funcionários de uma mineradora e verificar os fatores associados à pressão arterial elevada.

No presente estudo, o excesso de peso (sobrepeso + obesidade) foi observado em $76,1 \%$ da amostra (Tabela 2), o que é superior ao evidenciado no Brasil (INSTITUTO..., 2010b), bem como em funcionários da indústria alimentícia (QUEIROGA et al., 2009; CASSANI et al., 2009) e de empresa metalúrgica e siderúrgica (MARTINEZ; LATORRE, 2006), bem como de seguro de saúde (MOLENA-FERNANDES et al., 2006), porém inferior ao observado em motoristas profissionais (CAVAGIONI; PIERIN, 2010). Entre os sexos, a prevalência de sobrepeso e obesidade não diferiu, o que diverge do observado em outras profissóes

Tabela 1. Características descritivas da amostra por sexo.

\begin{tabular}{cccc}
\hline Variáveis & Homens $(\mathbf{n}=\mathbf{1 2 1})$ & Mulheres $(\mathbf{n}=\mathbf{7 6})$ & Total $(\mathbf{n}=\mathbf{1 9 7})$ \\
\hline Idade $(\mathrm{anos})^{*}$ & $40,1 \pm 9,0$ & $34,4 \pm 8,1$ & $38,08 \pm 9,0$ \\
IMC $\left(\mathrm{kg} \cdot \mathrm{m}^{-2}\right)^{*}$ & $28,80 \pm 4,2$ & $27,18 \pm 4,5$ & $28,18 \pm 4,4$ \\
PAS $(\mathrm{mmHg})^{*}$ & $125,5 \pm 19,1$ & $113,3 \pm 16,1$ & $121,28 \pm 18,9$ \\
PAD $(\mathrm{mmHg})^{*}$ & $78,9 \pm 12,1$ & $72,6 \pm 12,6$ & $76,71 \pm 12,5$ \\
PAM $(\mathrm{mmHg})^{*}$ & $94,4 \pm 14,0$ & $82,6 \pm 13,2$ & $91,56 \pm 14,2$ \\
\hline
\end{tabular}

IMC = índice de massa corporal; PAS = pressão arterial sistólica; PAD = pressão arterial diastólica; PAM = pressão arterial média; $*$ diferença $(\mathrm{p}<0,05)$ entre os sexos.

Tabela 2. Distribuição (n), prevalência (\%) e intervalos de confiança (IC), por sexo e para a amostra total, quanto à classificação do índice de massa corporal (IMC) e pressão arterial (PA).

\begin{tabular}{|c|c|c|c|c|c|c|c|c|c|}
\hline \multirow{2}{*}{ Variáveis } & \multicolumn{3}{|c|}{ Homens $(n=121)$} & \multicolumn{3}{|c|}{ Mulheres $(n=76)$} & \multicolumn{3}{|c|}{ Total $(n=197)$} \\
\hline & $\mathrm{n}$ & $\%$ & $(\mathrm{IC} 95 \%)$ & $\mathrm{n}$ & $\%$ & $(\mathrm{IC} 95 \%)$ & $\mathrm{n}$ & $\%$ & $(\mathrm{IC} 95 \%)$ \\
\hline \multicolumn{10}{|l|}{ IMC } \\
\hline Sobrepeso & 60 & 49,6 & $(48,6-50,6)$ & 31 & 40,8 & $(39,8-41,8)$ & 91 & 46,2 & $(45,2-47,2)$ \\
\hline Obesidade & 40 & 33,1 & $(32,2-34,0)$ & 19 & 25,0 & $(24,1-25,8)$ & 59 & 29,9 & $(29,0-30,8)$ \\
\hline \multicolumn{10}{|l|}{ PA } \\
\hline P. Hipertenso* & 49 & 40,5 & $(39,5-41,5)$ & 18 & 23,7 & $(22,9-24,5)$ & 67 & 34,0 & $(33,1-34,9)$ \\
\hline Hipertenso & 18 & 14,9 & $(14,2-15,6)$ & 7 & 9,2 & $(8,6-9,8)$ & 25 & 12,7 & $(12,0-13,3)$ \\
\hline
\end{tabular}

P. $=$ Pré; $*$ diferença entre os sexos $(\mathrm{p}<0,05)$. 
Tabela 3. Associação do sexo, idade e índice de massa corporal (IMC) com a pressão arterial (PA) elevada.

\begin{tabular}{|c|c|c|c|c|}
\hline \multirow{3}{*}{ Variáveis } & \multicolumn{4}{|c|}{ PA elevada } \\
\hline & \multirow{2}{*}{$\mathbf{n}$} & \multirow{2}{*}{$\%$} & Análise bruta & Análise ajustada ${ }^{b}$ \\
\hline & & & RP (IC 95\%) & RP (IC 95\%) \\
\hline \multicolumn{5}{|l|}{ Sexo } \\
\hline Feminino & 76 & 38,6 & 1 & 1 \\
\hline Masculino & 121 & 61,4 & $1,17(1,06-1,29)^{*}$ & $1,11(1,01-1,23)^{*}$ \\
\hline \multicolumn{5}{|l|}{ Idade } \\
\hline$\leq 40$ anos & 120 & 60,9 & 1 & 1 \\
\hline$>40$ anos & 77 & 39,1 & $1,17(1,07-1,28)^{*}$ & $1,13(1,03-1,25)^{*}$ \\
\hline \multicolumn{5}{|l|}{ IMC } \\
\hline Eutrófico & 47 & 23,9 & 1 & 1 \\
\hline Excesso de peso & 150 & 76,1 & $1,17(1,04-1,31)^{*}$ & $1,13(1,01-1,27)^{*}$ \\
\hline
\end{tabular}

$\mathrm{n}=$ frequência; $\%=$ prevalência; $\mathrm{RP}=$ razão de prevalência; ${ }^{\mathrm{b}}=$ ajustado para as demais variáveis da tabela; $*$ significativo $(\mathrm{p}<0,05)$.

(QUEIROGA et al., 2009; CASSANI et al., 2009; MOLENA-FERNANDES et al., 2006), em que a maior prevalência é observada nos homens. Frente a isso, parece que a profissão de minerador predispóe, em relação às demais, ao aumento da adiposidade corporal, e que esse efeito é semelhante em ambos os sexos.

Os valores pressóricos elevados (pré-hipertenso + hipertenso) foram observados em $46,1 \%$ dos voluntários (Tabela 2), o que é superior ao evidenciado em professores universitários (MOREIRA et al., 2011) e trabalhadores de empresa metalúrgica e siderúrgica (MARTINEZ; LATORRE, 2006), porém inferior ao encontrado em industriais (CASSANI et al., 2009). Em relação aos valores compatíveis com hipertensão arterial, a prevalência (12,7\%) (Tabela 2) foi inferior à observada no Brasil (SOCIEDADE..., 2010), bem como em motoristas profissionais (CAVAGIONI; PIERIN, 2010) e funcionários de empresa de seguro-saúde (MOLENA-FERNANDES et al., 2006). Sendo assim, em profissionais mineradores, $\mathrm{o}$ acometimento pela pressão arterial elevada parece ser equivalente ao de outras profissóes.

A prevalência de pré-hipertensão (Tabela 2) e a probabilidade de ter valores pressóricos elevados (Tabela 3) foram maiores nos homens, tal como evidenciado em outras profissões (MARTINEZ; LATORRE, 2006; MOREIRA et al., 2011; QUEIROGA et al., 2009; MOLENAFERNANDES et al., 2006). Dessa forma, parece que independente da atividade profissional, os homens são mais vulneráveis à instalação desse fator de risco cardiovascular, o que sugere a necessidade de priorizar as medidas preventivas a esse sexo.
A idade associa-se positivamente com a pressão arterial sistólica (WANG et al., 2006). No presente estudo, a idade superior a 40 anos associou-se, independente do sexo e da adiposidade corporal, à pressão arterial elevada (Tabela 3). Essa mesma relação foi evidenciada em professores universitários (MOREIRA et al., 2011) e em trabalhadores de empresa metalúrgica e siderúrgica (MARTINEZ; LATORRE, 2006). Portanto, as medidas preventivas contra a hipertensão arterial devem ser direcionadas àqueles com idade mais avançada, haja vista a imutabilidade desse fator de risco.

Com o aumento da adiposidade corporal há uma compilação de desordens neuroendócrinas que promovem a elevação dos valores pressóricos (KOTSIS et al., 2010). Em contrapartida, para cada quilo de massa corporal perdido, há redução de 1 mmHg na pressão arterial (NETER et al., 2003). No presente estudo, o excesso de peso associou-se à pressão arterial elevada, independente do sexo e idade (Tabela 3), tal como em outras profissóes (MARTINEZ; LATORRE, 2006; MOREIRA et al., 2011). Assim, a prevençáo e o controle do excesso de peso devem ser umas das principais medidas terapêuticas contra a hipertensão arterial, haja vista essa ser um fator de risco mutável e independente.

Como limitação, o presente estudo apresenta a não investigação dos hábitos alimentares (WANG et al., 2006; MARTINEZ; LATORRE, 2006), bem como dos níveis de estresse (MARTINEZ; LATORRE, 2006), os quais estáo relacionados aos valores pressóricos e poderiam alterar as associaçóes aqui observadas. Outra limitação é o fato das mensuraçóes dos valores pressóricos não caracterizarem diagnóstico clínico de hipertensão arterial, uma vez que tem sido indicada a realização de duas ou mais medidas 
(CHOBANIAN et al., 2003). Além disso, o fato de não ter sido analisado o nível de atividade física configura em uma importante limitação, à medida que essa variável parece predizer melhor a hipertensão arterial sistêmica do que o excesso de peso (MKHONTO et al., 2012). Apesar disso, esse é o primeiro estudo a trazer informaçôes sobre as condiçôes de saúde dos profissionais de mineração. Ademais, os resultados aqui obtidos poderão ajudar a nortear na elaboraçáo e direcionamento das medidas preventivas contra a hipertensão arterial e o excesso de peso nesse grupo de profissionais.

\section{Conclusão}

O excesso de peso e os valores pressóricos elevados foram observados em $76,1 \%$ e $46,1 \%$ da amostra, respectivamente, sendo a prevalência de pré-hipertensos maior nos homens. Ademais, a pressão arterial elevada associou-se ao sexo masculino, a idade superior a 40 anos e ao excesso de peso. Portanto, uma parcela expressiva dos mineradores encontra-se em risco para o desenvolvimento de doenças cardiovasculares e as medidas contra a hipertensão arterial devem ser direcionadas aos funcionários do sexo masculino e aqueles com idade superior a 40 anos, sendo a prevençáo e o controle do excesso de peso as principais medidas terapêuticas a serem adotadas.

\section{Agradecimentos}

À Coordenação de Aperfeiçoamento de Pessoal de Nível Superior (Capes) e ao Conselho Nacional de Desenvolvimento Científico (CNPq) pela concessão de bolsas de estudo em nível de iniciação científica (CNPq), mestrado (Capes), doutorado (Capes/ $\mathrm{CNPq}$ ) e de produtividade (CNPq).

\section{Referências}

BRAY, G. A. Medical consequences of obesity. Journal of Clinical Endocrinology \& Metabolism, Chevy Chase, v. 89, n. 6, p. 2583-2589, 2004.

BRASIL. Mnistério da Saúde. Conselho Nacional de Saúde do Brasil. Resolução no 196, de 10 de outubro de 1996. Aprovar as seguintes diretrizes e normas regulamentadoras de pesquisas envolvendo seres humanos. Diário Oficial da República Federativa do Brasil, Brasília, DF, 11 out. 1996. Seção 1. Disponível em: <http://conselho.saude. gov.br>. Acesso em: 25 abr. 2012.

CASSANI, R. S. L. et al. prevalência de fatores de risco cardiovascular em trabalhadores de uma indústria brasileira. Arquivos Brasileiros de Cardiologia, São Paulo, v. 92, n. 1, p. 16-22, 2009. http://dx.doi.org/10.1590/ S0066-782X2009000100004
CAVAGIONI, L. C.; PIERIN, A. M. G. Hipertensão arterial e obesidade em motoristas profissionais de transporte de cargas. Acta Paulista de Enfermagem, São Paulo, v. 23, n. 4, p. 455-460, 2010.

CHOBANIAN, A. V. et al. The National High Blood Pressure Education Program Coordinating Committee: the seventh report of the Joint National Committee on Prevention, Detection, Evaluation, and Treatment of High Blood Pressure: the JNC 7 report. Journal of the American Medical Association, Chicago, v. 289, p. 2560 2572, 2003. PMid:12748199. http://dx.doi.org/10.1001/ jama.289.19.2560

INSTITUTO BRASILEIRO DE GEOGRAFIA E ESTATÍSTICA - IBGE. Censo populacional de 2010. Rio de Janeiro: IBGE, 2010a. Disponível em: <http:// www.ibge.gov.br/cidadesat/topwindow.htm?1>. Acesso em: 25 abr. 2012.

INSTITUTO BRASILEIRO DE GEOGRAFIA E ESTATÍSTICA - IBGE. Pesquisa de orçamentos familiares 2008-2009: despesas, rendimentos e condiçôes de vida. Rio de Janeiro: IBGE, 2010b. Disponível em: <http://www. ibge.gov.br/home/estatistica/populacao/condicaodevida/ pof/2008_2009/POFpublicacao.pdf>. Acesso em: 25 abr. 2012 .

I N S T I T U T O B R A S I E I R O D E MINERAÇÃO - IBRAM. Informações e Análises da Economia Mineral Brasileira. 6. ed. Brasília: IBRAM, 2011. 7 p. Disponível em: <http://www.ibram.org.br/ sites/1300/1382/00001418.pdf>. Acesso em: 25 abr. 2012.

KOTSIS, V. et al. Mechanisms of obesity-induced hypertension. Hypertension Research, Toyonaka, v. 33, p. 386-393, 2010.

LAHTI-KOSKI, M. et al. Associations of body mass index and obesity with physical activity, food choices, alcohol intake, and smoking in the 1982-1997 FINRISK Studies. American Journal of Clinical Nutrition, Bethesda, v. 75, p. 809-817, 2002.

LEWINGTON, S. et al. Age-specific relevance of usual blood pressure to vascular mortality: a meta-analysis of individual data for one million adults in 61 prospective studies. Lancet, London, v. 360, n. 9349, p. 1903-1913, 2002. http://dx.doi.org/10.1016/S0140-6736(02)11911-8 MARTINEZ, M. C.; LATORRE, M. R. D. O. Fatores de risco para hipertensáo arterial e diabete melito em trabalhadores de empresa metalúrgica e siderúrgica. Arquivos Brasileiros de Cardiologia, São Paulo, v. 87, p. 471-479, 2006. http://dx.doi.org/10.1590/ S0066-782X2006001700012

MKHONTO, S. S. et al. Association of body weight and physical activity with blood pressure in a rural population in the Dikgale village of Limpopo Province in South Africa. BMC Research Notes, London, v. 5, n. 118, p. 118, 2012 PMid:22361366 PMCid:3392726. http://dx.doi. org/10.1186/1756-0500-5-118

MOLENA-FERNANDES, C. A. et al. Associação entre sobrepeso e obesidade e fatores de risco cardiovascular em funcionários de uma empresa de seguro-saúde. Revista da Educação Física/UEM, Maringá, v. 17, n. 1, p. 75-83, 2006. 
MOREIRA, O. C. et al. Associação entre risco cardiovascular e hipertensão arterial em professores universitários. Revista Brasileira de Educação Física e Esporte, v. 25, n. 3, p. 397-406, 2011.

NETER, J. E. et al. Influence of weight reduction on blood pressure: a meta-analysis of randomized controlled trials. Hypertension, Dallas, v. 42, n. 5, p. 878-884, 2003. PMid:12975389. http://dx.doi.org/10.1161/01. HYP.0000094221.86888.AE

ORGANIZAÇÃO MUNDIAL DA SAÚDE - OMS. Physical Status: The Use and Interpretation of Anthropometry. Genebra: OMS, 1995. (Technical Report Series, n. 854).

PAN, S. Y. et al. Association of obesity and cancer risk in Canada. American Journal of Epidemiology, Baltimore, v. 159, n. 3, p. 259-268, 2004. http://dx.doi.org/10.1093/ aje/kwh041

POPKIN, B. M.; DOAK, C. The obesity epidemic is a worldwide phenomenon. Nutrition Reviews, Washington, v. 56, p. 106-114, 1998. http://dx.doi. org/10.1111/j.1753-4887.1998.tb01722.x

PROGRAMA DAS NAÇÓES UNIDAS PARA O DESENVOLVIMENTO. Ranking do indice de desenvolvimento municipal dos municipios do Brasil. Brasília: PNUD, 2012. Disponível em: <http://www.pnud. org.br/atlas/tabelas/index.php>. Acesso em: 25 abr. 2012. QUEIROGA, M. R. et al. Efeitos da idade e dos indicadores de obesidade na pressáo arterial de trabalhadores. Motriz: revista de educação física, Rio Claro, v. 15, n. 3, p. 631-640, 2009.

RANKINEN, T. et al. The human obesity gene map: the 2005 update. Obesity (Silver Spring), Silver Spring, v. 14, n. 4, p. 529-644, 2006.

SOCIEDADE BRASILEIRA DE CARDIOLOGIA - SBC. VI Diretrizes Brasileiras de Hipertensão. Arquivos Brasileiros de Cardiologia, São Paulo, v. 95, n. 1, p. 1-51, 2010.

VERMA, A. et al. Hypothyroidism and obesity. Cause or effect? Saudi Medical Journal, Riyadh, v. 29, n. 8, p. $1135-1138,2008$

WANG, W. et al. A Longitudinal Study of hypertension risk factors and their relation to cardiovascular disease:The Strong Heart Study. Hypertension, Dallas, v. 47, p. 403-409, 2006. http://dx.doi.org/10.1161/01. HYP.0000200710.29498.80

\section{Contribuição dos Autores}

Marcelo Magalháes Sales: responsável pela concepção, análise e interpretação dos resultados obtidos e redação do manuscrito e revisão final. Thales Boaventura Rachid Nascimento: responsável pela concepção, análise e interpretação dos resultados e redação do manuscrito. Ricardo Yukio Asano: responsável pela concepção e redação do manuscrito. José Fernando Vila Nova de Moraes: auxiliou na análise e interpretação dos resultados, bem como participou na redaçáo do manuscrito. Rafael da Costa Sotero: auxiliou na redação do manuscrito. Verusca Najara de Carvalho Cunha: auxiliou na análise e interpretaçáo dos resultados e também na confecção do manuscrito. Rodrigo Alberto Vieira Browne: pesquisa bibliográfica e redação do manuscrito. Herbert Gustavo Simóes: responsável pela concepção, redação do manuscrito e revisão final.

\section{Notas}

${ }^{1}$ Os resultados do presente trabalho são parte de pesquisa envolvendo seres humanos e os procedimentos éticos vigentes foram cumpridos conforme Resolução 196/96 do Conselho Nacional de Saúde do Brasil. 